\section{ENCUENTROS Y DESENCUENTROS CON LA FÓRMULA DEL RIESGO Y LOS DESASTRES: TIEMPOS PARA EL CUIDADO Y LA RECIPROCIDAD}

Ma. Teresa Armijos Burneo ${ }^{{ }^{*}}$ y Viviana Ramírez Loaiza ${ }^{2}$

\section{RESUMEN}

La fórmula 'Amenaza x Vulnerabilidad = Riesgo' ha sido fundamental en visibilizar, politizar y develar la construcción social del riesgo de desastres. Sin embargo, esta formula y críticas a la misma se han posicionado desde categorías del pensamiento occidental. A partir de los aprendizajes que hemos construido con mujeres embera pertenecientes al Cabildo urbano indígena Kurmadó (Risaralda, Colombia) este articulo propone una reflexión teórica y metodológica, desde una perspectiva crítica-feminista, considerando relaciones de saberes inspiradas por el pensamiento decolonial latinoamericano. Nos preguntamos: ¿qué pueden (o deben) aprender los estudios de riesgos y desastres de estas mujeres y de sus saberes del habitar el territorio? El primer encuentro es una lectura breve de la historia de los estudios de riesgos y desastres. El segundo encuentro, presenta la investigación co-producida con las mujeres embera y problematiza la gestión del riesgo en su sentido más socio-lingüístico, considerando las prácticas de cuidado hacia y desde sus cuerpos y sus territorios. En el encuentro tres, presentamos una reflexión a partir de la geografía critica y el pensamiento decolonial. Finalmente, hacemos un llamado para que los estudios de desastres reconozcan epistemologías y saberes desde otras formas de vivir y dar respuesta al riesgo en America Latina.

\section{PALABRAS CLAVES}

Territorio, Pensamiento decolonial, Cuidado, Reciprocidad, Riesgo de desastres

\section{ENCOUNTERS AND (DIS) ENCOUNTERS WITH THE RISK AND DISASTER FORMULA: TIMES FOR CARE AND RECIPROCITY}

\section{ABSTRACT}

The formula 'Hazard $\mathrm{x}$ Vulnerability = Risk' has been central in making visible, politicizing, and revealing the social construction of disaster risk. However, this formula and its criticisms are based on Western categories of knowledge production. Based on what we have learnt through our work with Embera women belonging to the Kurmadó Indigenous Council (Risaralda, Colombia), this article proposes a theoretical and methodological reflection, from a critical-feminist perspective inspired by Latin-American decolonial thought. We ask: what can (or should) the studies of risk and disasters learn from these women and from their ways of inhabiting the territory? The first section presents a summary of the history of risk and disaster studies. The second section presents the research co-produced with the Embera women and problematizes risk management in its most socio-linguistic sense, considering their practices of care towards and from their bodies and their territories. The final section presents a reflection based on critical geography and decolonial thinking. We therefore invite disaster studies to recognise epistemologies and knowledges based on other ways of experiencing and responding to risk in Latin America.

\author{
1. Departamento de \\ Desarrollo Internacional, \\ Universidad de East Anglia, \\ Norwich, Reino Unido. \\ 2. Observatorio Psicosocial \\ para la Gestión del Riesgo \\ de Desastres, Universidad \\ de Manizales, Manizales, \\ Colombia. \\ *Autor de correspondencia: \\ t.armijos@uea.ac.uk
}

Identificador:

http://revistareder.com/

handle-0719-8477-2021-095

\section{RECIBIDO}

11 de febrero de 2021

ACEPTADO

19 de mayo de 2021

\section{PUBLICADO}

1 de julio de 2021

Formato cita

Recomendada (APA):

Armijos Burneo, M.T. \& Ramírez Loaiza, V. (2021). Encuentros y Desencuentros con la Fórmula del Riesgo y los Desastres: Tiempos para el Cuidado y la Reciprocidad. Revista de Estudios

Latinoamericanos sobre Reducción del Riesgo de Desastres REDER, 5(2), 1933. http://revistareder.com/ handle-0719-8477-2021-095

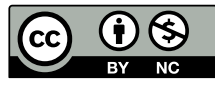

Todos los artículos publicados en REDER siguen una política de Acceso

Abierto y se respaldan en una Licencia CreativeCommons Atribución-NoComercial 4.0 Internacional.

Revista de Estudios

Latinoamericanos sobre Reducción del Riesgo de Desastres (REDER)

\title{
KEYWORDS
}

Territory, Decolonial thinking, Care, Reciprocity, Disaster risk 


\section{INTRODUCCIÓN}

Nuestra permanencia en los estudios de riesgo y desastres se ha encontrado de la mano de comunidades, muchas de ellas olvidadas por lo que denominamos como planeación territorial y justicia social. Aprendizajes colectivos sobre el territorio, sentires e ideas, definiciones y teorías sobre lo que parece ser la dialéctica entre el riesgo y el cuidado, nos ha permitido empezar a caminar con otras sospechas, muchas de ellas propiciadas por las preguntas y proyectos de las personas y comunidades con las que trabajamos y quienes diariamente afrontan múltiples riesgos. Estas experiencias han puesto en evidencia algunos límites de las herramientas teóricas y metodológicas de los estudios de riesgos y por ende de la gestión del riesgo de desastres, exigiéndonos buscar respuestas y prácticas desde otras categorías de saber-ser las cuales implican la valoración de epistemologías y ontologías plurales.

Entre los vínculos generados durante y después de los proyectos, nos situamos en el proyecto IdentificArte "Movilizándonos con el riesgo: desplazamiento forzado y vulnerabilidad en zonas de riesgo en Colombia"'. En este proyecto trabajamos con un grupo de mujeres embera pertenecientes al Cabildo urbano indígena Kurmadó de la región de Risaralda, Colombia. A través de los círculos de la palabra, conversaciones alrededor del alimento, danza y expresiones creativas, en el marco del pensamiento decolonial, este artículo ofrece algunas reflexiones en relación a la pregunta ¿qué puede (o debe) aprender los estudios de riesgos y desastres de estas mujeres y de sus saberes del habitar el territorio?

Esta pregunta no solo convoca los aprendizajes construidos alrededor del significado de habitar desde los cuerpos de estas mujeres, además, evoca la reflexividad de las investigadoras, mujeres mestizas-latinoamericanas, quienes en la búsqueda de un ejercicio ético de investigación, se encuentran con el conocimiento situado (Haraway, 1991) y las metodologías de investigación acción participativas (Fals-Borda, 2008). Bajo la idea que el sujeto investigador transita a ser el sujeto investigado (Cruz, Reyes y Cornejo, 2012), convenimos dar inicio al artículo con unos relatos autobiográficos cortos que evoquen el lugar de entendimiento y de poder de las investigadoras. Posteriormente, narramos el primer encuentro basado en el diálogo sostenido con la reconocida y legítima fórmula de representar el riesgo de desastres $(A \times V=R)$, dando cuenta de la importancia que ha tenido esta fórmula para visibilizar el origen sociopolítico de una emergencia o un desastre, así como para lograr procesos de prevención, mitigación y conocimiento del riesgo.

El segundo encuentro, relata la experiencia sostenida con las mujeres embera en el marco del proyecto IdentificArte durante dos años. Esta experiencia motivó un giro importante en nuestra practica de investigación, a partir del cual "nuestro antiguo objeto (de investigación) sea hoy el que nos interpele, nos diga quien somos y que espera de nosotros y nos demande el uso de nuestra 'caja de herramientas' para responder sus preguntas y contribuir con su proyecto histórico." (Segato 2018b, p.14). Esto significó aprender de su forma de organización comunitaria, de rituales y expresiones en lengua propia que manifiestan su sentido de cuidado territorial, además de construir otros, nuevos, sentidos con las investigadoras (mestizas).

Caminamos hacia el tercer encuentro: la figura del giro decolonial ¿Por qué nos acercamos a esta forma de pensamiento y de posición ética-política? Emergieron desencuentros entre lo que la fórmula y los estudios de riesgo y desastres nos planteaban sobre lo que eran fenómenos amenazantes, vulnerabilidades económicas, físicas y sociales, con aquellos hechos y situaciones que para las mujeres significaba amenazas, riesgo, cuidado y seguridad. Esto nos llevó a buscar otras categorías de saber que nos permitiera entender al territorio como elemento intrínseco del bienestar colectivo y del cuerpo. Bajo este 'desencuentro', traemos a Rita Segato (2018) y Catherine Walsh $(2013,2015)$ quienes nos han ayudado a forjar sentidos desde las categorías de 'colonialismo del saber' y 'pluralidad del saber-ser'. Una vez regresamos a la fórmula, encontramos límites comprensivos frente a las formas de comprender el territorio en cuanto al origen colonial que le antecede: la dualidad entre cuerpo y espacio (Zaragocin y Caretta, 2020). Así, a través de "(...) la perspectiva decolonial se propone reatar con lo que existe, con los jirones de comunidad que continúan su camino histórico" (Segato, 2018b, p.64).

Por último, proponemos la categoría ontológica y metodológica que impacta directamente los abordajes y métodos de investigación con las comunidades indígenas: la reciprocidad. Esta categoría responde a un camino ético que se traza desde el cuidado entre los cuerpos como territorios y nuestros cuerpos como investigadoras, ubicados en el encuentro de la conversación; en
1. El proyecto Identificarte, con nombre en Inglés Moving with Risk: Forced Displacement and vulnerability to hazards in Colombia, fue financiado por el fondo Global Challenges Research Fund (GCRF) del consejo de Investigación del Reino Unido (UKRI) entre noviembre 2016 y enero de 2019. Además, se realizó una reflexión metodológica junto con las mujeres y hombres embera que participaron en el proyecto IdentificArte a través de la convocatoria Indigenous Engagement, Research Partnerships and Knowledge Mobilisation, financiado por Arts and Humanities Research Council (AHRC) del Reino Unido entre el 2018 y 2019. 
este sentido, entendemos que este diálogo está atravesado por la figura de mutualidad, mutualidad de aprendizajes, historias y saberes, que terminan configurando espacios de cuidado del cuerpoterritorio. El cuidado, en este sentido, no solo se visibiliza desde un plano metodológico en términos de los vínculos tejidos entre co-investigadoras (investigadoras y participantes), también se retrata en el plano epistémico y ontológico de la forma como se habitan los territorios.

\section{UNA HISTORIA BREVE: RELATOS AUTOBIOGRÁFICOS EN REFLEJO}

Ante la materialidad de la injusticia que se vive en los países de dónde venimos, empezamos en la búsqueda de apegarnos a la esperanza. Ma. Teresa, mujer que nace en Ecuador a principios de los años 80, desde temprana edad, se cuestiona la desigualdad. Encuentra en la historia y las ciencias sociales caminos para hacer sentido a esas realidades que la rodean, para saber reconocer las luchas de poder y sobre todo, para aprender de las experiencias de otros. Tras sus estudios, investigaciones y varias estadías en diferentes países de América Latina, llega a los estudios de riesgo y desastres. A partir de una lectura desde la ecología política (Wisner et. al., 2004) se encuentra con historias de vida atravesadas por la desigualdad social pero a la vez, con un sentido permanente de resistencia. Es docente e investigadora dedicada a visibilizar esas historias y a generar procesos de investigación acción que promuevan el intercambio de saberes.

Viviana, nace a principios de los 90 en Colombia. Le ampara estar en una ciudad que, aparentemente, no es afectada por el conflicto armado, o que no tiene las manifestaciones de violencia que se presentan en otras zonas del país, y que Viviana sólo conoce a través de las noticias. Sin embargo, años después, se da cuenta que hay varias historias intergeneracionales detrás que le acompañan, o por los menos dos. El Peñol -lugar de nacimiento de su padre- fue inundado por un proyecto hidroeléctrico que llevó a que su familia paterna fuera despojada. Historia dos: uno de sus tíos fue secuestrado y asesinado por una de las guerrillas de Colombia. De manera coincidencial, la reducción del riesgo de desastres y los estudios de conflicto armado, forjaron sus sentidos para la disciplina psicológica. Ahora se encuentra como consultora e investigadora en proyectos que acompañen y fortalezcan procesos comunitarios con víctimas del conflicto armado.

Así, bajo la idea de Davis (2015, p.121) - "caminar el paisaje es involucrarse en un gesto permanente de afirmación" -, las co-autoras andan las realidades de América Latina, limitadamente narradas hasta hace poco tiempo, para afirmarse desde la inquietud y la sospecha con el trabajo comunitario y las metodologías participativas en la reducción del riesgo de desastres. El proyecto STREVA (Fortalecimiento de la Resiliencia en Zonas Volcánicas- www.streva.ac.uk) se convierte en el encuentro y camino inicial entre ambas autoras en los estudios de riesgo y desastres. Bajo este contexto y regresando a nuestra narrativa en primera persona, buscamos que este artículo de testimonio de las interpelaciones que nos han permitido construir y deconstruir marcos epistémicos, ontológicos y metodológicos. Iniciamos con aquellos hitos teóricos que nos motivaron a profundizar en el campo de los estudio de riesgos y desastres, y centrarnos en el conocimiento decolonial y la reducción del riesgo como apuesta ética y política.

\section{Primer encuentro: estudios de riesgos y desastres}

En un inicio, encontrar la fórmula para representar el riesgo propuesta desde el enfoque de las ciencias aplicadas (Amenaza $x$ Vulnerabilidad = Riesgo) (Maskrey, 1998), y los análisis realizados alrededor de ella, nos permitió visibilizar el rol de las ciencias sociales con mayor claridad en el campo de la gestión del riesgo de desastres, al determinar que el riesgo, como construcción social, involucra factores sociales, económicos y políticos que consolidan la vulnerabilidad. Para Maskrey (1998, p.13), transitar del enfoque de las ciencias naturales a las ciencias aplicadas a través de esta fórmula, "representó un cambio en el énfasis del estudio de las amenazas hacia el estudio de sus impactos y efectos".

Entre los estudios derivados para latinoamérica, se encuentra Narvaez, Lavell y Pérez (2009) quienes analizaron la gestión del riesgo desde el enfoque de procesos, enfatizando en la importancia de estudiar y definir aquellos riesgos futuros -prospectivos-, los riesgos mitigables -correctivos- y aquellos ya materializados en emergencias y desastres -reactivos- en un territorio.

Las publicaciones realizadas por LA RED (Red de Estudios Sociales en Prevención de Desastres en América Latina) también brindaron una solidez conceptual desde las ciencias sociales a la gestión del riesgo de desastres. Si bien estas publicaciones se dieron hace más de 17 años, traemos algunas comprensiones que nos permitieron tejer puentes con otras disciplinas de las ciencias sociales, e inclusive con el giro decolonial: 
1. "El desastre visto como un proceso iniciado mucho antes de su detonación, probablemente se parece mucho más a la sociedad que lo sufre que a la naturaleza" (LA RED, 1994, p.6). El desastre entonces, resulta ser una equivocación en la concepción e implementación de los proyectos de desarrollo, y a su vez una oportunidad de reconstrucción ética, política, económica y social para el territorio.

2. En términos filosóficos, estudiar el riesgo y su reducción, es analizar las representaciones del tiempo y su función en la sociedad, especialmente el tiempo futuro que se convierte en un tiempo no-existente, lo que trae mayor dificultad en materializar acciones ante un posible evento, más aún, dentro de una visión cortoplacista (Evans, 1994).

3. La cotidianidad se convierte en soporte de la acción ante una emergencia (LA RED, 1993, p.5) y de los impactos generados por aquellas emergencias que parecen ser naturalizadas ante la alta frecuencia con la que ocurren, y que eran ignorados inicialmente por los propósitos trazados para el desarrollo sostenible (Bender, 1993). Por lo tanto, "los desastres, en realidad, están más cerca de la vida cotidiana de lo que la cultura formal quisiera. Ellos son parte de la vida y la vida siempre, la que existía antes y después, tiene un lugar en ellos" (LA RED, 1994, p.6).

4. La vulnerabilidad como categoría articuladora en los estudios de desastre, permitió redefinirse desde el enfoque de las ciencias sociales como "una medida relativa de la capacidad de una población de absorber y recuperarse de un daño o pérdida determinada" (Maskrey, 1998, p.15-16). En esta línea, se dan reflexiones en torno a la capacidad local y a las estrategias de gestión de riesgos, centrándose en disminuir la exposición a las amenazas y en la reducción de las vulnerabilidades (Wilches-Chaux, 1993).

Estos primeros antecedentes se articularon con estudios derivados de la fórmula y el papel de las ciencias sociales y humanas en el campo de la gestión del riesgo de desastres. Uno de ellos fue el conocido texto de Wisner, Blaikie, Cannon y Davis $(1996,2004)$ donde, bajo un análisis crítico hacia la vulnerabilidad, los autores proponen el modelo de 'modelo de presión y liberación', involucrando vulnerabilidades más estructurales con condiciones especificas de inseguridad. Maskrey (1998, p.22), por su parte, propuso un enfoque holístico para visibilizar de nuevo el estudio de la vulnerabilidad en relación con las amenazas, estableciendo los escenarios de riesgos, los cuales se construyen en el "encuentro de procesos, tanto naturales como sociales, con las unidades sociales y sus estrategias de gestión", y que permiten entender la medida de la escala fractal del riesgo (cambios drásticos en espacios limitados y heterogéneos).

Tuner et al. (2003) se enfocaron en analizar la vulnerabilidad como un sistema complejo lo que permite incluir y teorizar categorías como la capacidad de respuesta y la exposición hacia el peligro. Al reconocer los límites de la fórmula, estos autores proponen una fórmula que de cuenta de las interacciones y las reestructuraciones que surgen después de una emergencia o desastre (Riesgo = perturbación, exposición al peligro, capacidad del sistema para manejar el shock).

Este texto clave, fue antecedido por algunas tesis de Gustavo Wilches-Chaux, quien era miembro de LA RED. Su teorización sobre la vulnerabilidad global (1993) desde un análisis dinámico de sistemas, permitió diferenciar conceptualmente elementos clave de la construcción de vulnerabilidad y sus implicancias en acciones de mitigación. Posteriormente, planteó varías críticas frente a la poca legitimidad que tienen las construcciones comunitarias en el campo de estudio de riesgo y desastres, y la separación reiterada entre la vida humana y la vida de los ecosistemas. Wilches-Chaux (2017) posiciona entonces el concepto-herramienta seguridad territorial por medio del cual relaciona el riesgo y protección en clave de las formas de prevención que nacen desde prácticas culturales propias de la construcción territorial. En complemento, Virginia García (2017), desde la antropología de los desastres, mostró la complejidad de la relación entre naturaleza y cultura en medio de marcos históricos que dan algunas respuestas frente a la manera como se viven los fenómenos naturales en diferentes comunidades. En consonancia, esta autora enfatiza "La construcción social de riesgos remite a la producción y reproducción de las condiciones de vulnerabilidad que definen y determinan la magnitud de los efectos ante la presencia de una amenaza natural; es por ello la principal responsable de los procesos de desastre." (García, 2005, p.23). 
Así, entre el enfoque de las ciencias aplicadas y las ciencias sociales, logramos una primera comprensión, ubicando las vulnerabilidades y las capacidades como marcos clave de entendimiento y acción en el campo de los estudios de riesgo y desastres.

\section{Encuentro 2: Proyecto IdentificArte, entre la gestión de riesgos y otras formas de ser-estar en el territorio}

El proyecto IdentificArte "Movilizándonos con el Riesgo: Desplazamiento y Vulnerabilidad en Zonas de Riesgo de Desastres en Colombia" tuvo tres objetivos; el primero, se enfocó en comprender las trayectorias de riesgo, recursos y vulnerabilidades de las personas que vivieron el desplazamiento forzado y que se encuentran viviendo en zonas de riesgo de desastres. El segundo, se basó en el pilotaje de una metodología basada en las artes como estrategia para la reflexión y apertura de espacios de diálogo de saberes, y a su vez, enfocada en aportar a cambios a nivel individual y colectivo. Finalmente, el tercer objetivo buscó el fortalecimiento de las capacidades individuales, comunitarias e institucionales en gestión del riesgo de desastres.

Si bien trabajamos con seis comunidades en diferentes localidades en Colombia (Few et al. 2021), los encuentros relatados y reflexionados en este artículo se enfocan en el trabajo que realizamos con las mujeres embera pertenecientes al Cabildo Indígena Kurmadó en Risaralda, Colombia. Trabajamos con 30 mujeres aproximadamente de edades entre 16 y 60 años. Estas mujeres habitan en territorios marginales excluidos geográfica y simbólicamente de la atención del Estado y las gobernabilidades locales. Han tenido que padecer los efectos del conflicto armado, desplazándose de manera forzada de sus territorios de origen.

El desplazamiento masivo de familias indígenas provenientes del Resguardo Unificado Chamí del municipio de Pueblo Rico y Mistrató empezó alrededor del año 2000. El contexto de conflicto armado representaba un riesgo para miles de familias que se encontraban confinadas ante enfrentamientos o recibían amenazas directas de los actores armados. Una participante enfatizaba que cuando era pequeña y vio el grupo armado llegar a su casa para usar su cocina y pernoctar, les decía:

"-oiga ustedes qué van a hacer con eso- y él me decía: -usted tiene que ser (tener) mucho cuidado de aquí en adelante, que aquí va haber una guerra (...) cuando ustedes escuchen un tiro no se van a salir pa' (para) afuera, sino que se quedan extendidos en el suelo (...) venía la guerra y nosotros pal monte." (Joven indígena, comunicación personal, 10 de octubre de 2018).

Es así como muchas de estas familias deciden partir del resguardo hacia el Municipio de Pereira en Risaralda. En el año 2000 se crea la Asociación Solidaria de Indígenas Desplazados y Vulnerables; en el 2002 la Asociación se unifica como Cabildo Mayor de Pereira y en el 2004 se posiciona como Cabildo Indígena Kurmadó: korma (piedra preciosa y chaquira) y do (río) (Altamirano y Sánchez, 2017).

Geográficamente, estas familias y particularmente las mujeres con las que trabajamos, vivieron y han vivido dinámicas de riesgo de desastres. Algunas áreas de las zonas en donde viven actualmente en el Municipio de Pereira se encuentran declaradas como zonas de alto riesgo; asociados a deslizamientos, inundaciones e incendios (Few et al. 2021).

Sin embargo, durante décadas estas mujeres han construido otras formas de habitar en medio de la marginalidad y la vulnerabilidad; han configurado nuevas identidades y prácticas culturales por medio de rituales, danzas tradicionales, venta de artesanías y algunos trabajos remunerados en la recolección de cosechas así como en limpieza de casas. Se han organizado desde la estructura indígena de gobierno y justicia propia. Desde esta gobernabilidad y formas de habitar el territorio, han respondido a diferentes tipos de riesgos y amenazas que enfrentan día a día, entre ellos, inundaciones, amenazas de bandas criminales y la discriminación cotidiana por parte de los habitantes mestizos.

La metodología de investigación acción con este grupo de mujeres se basó en cuatro momentos; en el primero, nos enfocamos en contactar a comunidades, líderes del cabildo Indígena, e instituciones para conversar y llegar a acuerdos frente al alcance del proyecto. En el segundo momento metodológico, evocamos conversaciones sobre historias autobiográficas y colectivas a partir de canciones y de la realización de artesanías propias con chaquiras, para realizar collares, 
pulseras, entre otros accesorios. De manera simultánea, algunas de ellas participaron en un proceso de formación junto con los participantes mestizos en la política de gestión del riesgo de desastres de Colombia y los conceptos clave que la comprenden.

Para el último momento, basándose en sus historias de vida, respuesta y recuperación ante el desplazamiento y los desastres, las mujeres diseñaron una serie de danzas tradicionales ${ }^{2}$ que fueron interpretadas en la plaza principal de la ciudad de Pereira, Colombia en septiembre de 2018. A este evento asistieron representantes de instituciones locales y nacionales, vecinos y personas de otras comunidades. A finales de ese mismo año, elaboramos con varias mujeres y líderes embera la sistematización metodológica de todo el proceso durante varios encuentros que incluyeron el círculo de la palabra y un ritual con el Jaibaná (médico tradicional embera) (Armijos, Ramírez y López, 2020).

Es justamente durante estas conversaciones, talleres, y presentaciones, que surgen desencuentros entre sus historias y experiencias con las herramientas teóricas de los estudios de riesgo y desastres. Para nosotras y el equipo del proyecto, estos desencuentros fueron conceptuales, epistemológicos, ontológicos y metodológicos, los cuales generaron varios espacios de reflexión y aprendizaje que presentamos a continuación.

\section{¿Quiénes o qué son los jais?}

El primer des-encuentro fue sociolingüístico. ¿Qué es riesgo? No hay traducción al embera. ¿Un peligro que puede suceder? "Pero si estos peligros solo son dados por los Jais", nos explicaron las mujeres con las que trabajamos (10 de octubre de 2018). Durante ese mismo encuentro, el gobernador del cabildo enfatizó que un jai es el dueño, el don de las sabidurías milenarias", el médico tradicional embera añadió:

"Cuando el río se crece o pasa un derrumbe por ahí, está un mal espíritu que dejó el Jaibaná guardado y cuando crece el río, lo destapa, y ahí sale. Cuando sale ese espíritu no es un espíritu bueno, es malo (...) guardamos los malos, por eso, porque son malos, si lo tenía guardado (un jai) en una raíz de un árbol muy grande cuando se da un vendaval, ahí sale el Jai." (Médico tradicional embera, 10 de Octubre de 2018).

En este sentido, "los riesgos" se dan por la presencia de espíritus Jai negativos. Si "los riesgos" se materializan, hay emergencia: el territorio se enferma. No obstante, los responsables y aquellos que están en la capacidad de llevar a cabo la sanación del territorio son los Jaibanás, quiénes a partir del poder espiritual, llevan a cabo rituales para salvaguardar y recuperar la vida, la subsistencia y la naturaleza. Por tanto, un jai negativo, que también se puede manifestar en animales muertos o enfermos, es retirado de un territorio mediante un Jai positivo creado por un Jaibaná, "(...) pero cantando Jaibaná, mirando la tierra con espíritu para poder cerrar lo que estaba andando la calle (el) animal." (Conversación grupal Caimalito, 14 de agosto de 2017).

Este peligro, o la capacidad de generar daño por parte de los jais negativos, no se relaciona solamente con amenazas asociadas a fenómenos naturales ni está separado de los cuerpos o territorios donde estos habitan, como si el cuerpo estuviera en un lugar y la amenaza en otro sitio. Justamente hay una relación de unidad entre cuerpo, comunidad y territorio la cual, ante un determinado evento, tiene consecuencias directas en los tres. La 'enfermedad' o 'peligro' se manifiesta en el espacio geográfico y en el cuerpo al mismo tiempo.

"[...] afectaba mucho con la enfermedad, se empezó a enfermar niños que estaban alentaditos. Y así, dejaron ver Médico tradicional y nos decía, la tierra está dañada [...] (Joven indígena, comunicación personal, Pereira, 1 de noviembre de 2017).

En consecuencia, el cuerpo, su salud mental y física, es comunitaria y relacional; depende de los estados de curación y enfermedad del territorio que están al cuidado del Jaibaná y del conocimiento en plantas que cada familia y comunidad tenga: "si conocemos las plantas podemos curar y ayudar" (Jóven indígena, comunicación personal, Pereira, 1 de noviembre de 2017). En esta sentido, " (...) las plantas que están aquí son plantas medicinal, son seres vivos como nosotros, hoy esa planta está trabajando en pro de ustedes." (Gobernador Mayor, Cabildo Indígena Kurmadó, 27 de octubre 2018).
2. Para mayor información de la metodología véase https://youtu.be/ DYW9cfoJmh4 
Los procesos de respuesta ante el riesgo para la comunidad indígena embera implican el trabajo de cuidado a través de la medicina tradicional ya que la sanación del territorio es la sanación misma del cuerpo. Prevenir y responder a una emergencia o un desastre es también prevenir o responder a la enfermedad. El cuidado se practica a través de rituales dentro de la familia, del uso de plantas medicinales y también de los rituales con los Jaibanás. A continuación, mostramos una figura en la que sintetizamos nuestra interpretación, evocando que, la circularidad de estas comprensiones, emerge a partir de la movilización de Jais, propiciadas por un Jaibaná (ver Figura 1).

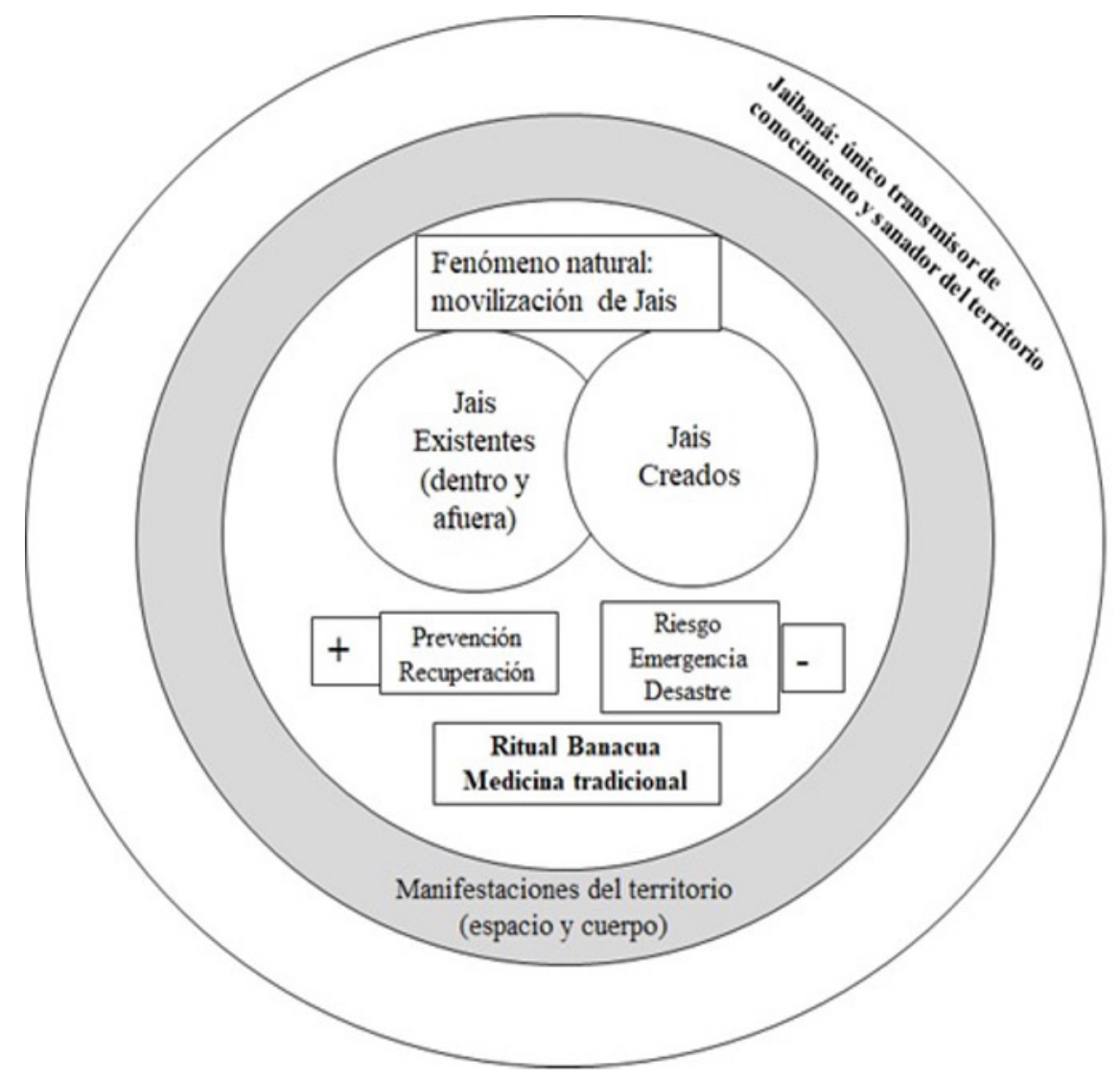

Figura 1. Cuidado del cuerpo-territorio

Fuente: Autoras, 2021

\section{Tiempos de escucha, tiempos de cuidado}

Nuestro segundo desencuentro importante durante el proyecto fue en el orden metodológico. A finales de 2017, después de varias visitas y conversaciones con líderes y lideresas comunitarios, empezamos el trabajo con las mujeres embera en dos comunidades cerca de la ciudad de Pereira. Dimos inicio con unas primeras conversaciones individuales. Al notar que con los mestizos la metodología de evocación con la música había sido tan impactante (Marsh, Armijos y Few, 2020), intentamos usar la misma estrategia, sin muchos resultados. Silencios y algunas risas provocaban esta manera de conversar. Nos dimos cuenta que, en algunas de las conversaciones individuales, a pesar de haber sido diseñadas a partir de metodologías participativas (Marsh et al. 2020) y con el propósito de propiciar diálogo con las mujeres embera, no abrían espacios de intercambio, y generaban momentos de incomodidad tanto para nosotras como para ellas.

Así, convenimos con ellas hacer un cambio y realizar conversaciones colectivas que nos permitiera escuchar sus historias en el territorio de origen, su llegada a los territorios en los que actualmente habitan y sus diferentes formas de respuesta ante sus trayectorias de vida. Realizamos conversaciones en grupo, generalmente en lengua embera, donde una o varias de ellas interpretaban la conversación. Dentro de estos espacios, hubo momentos en los que sus preguntas eran directas hacia nuestras vidas y nuestras formas de sentir con ellas "¿Cómo se sienten con nosotras?" "¿Usted tienen familia? La debe de ver poco por sus viajes". 
Los tiempos para los encuentros también fueron distintos. Era recurrente que los horarios de inicio y fin de las actividades se salieran de la planeación, o que nos cancelaran dos o un día antes el encuentro programado. Difícilmente empezábamos y terminábamos a la hora que habíamos planeado. Esto nos invitó a pensar que las metodologías de trabajo necesitaban recrear otros tiempos, tal vez plurales, y flexibles en los que se permita "el cambio de planes" permanentemente. Poco a poco fuimos entendiendo que el control sobre los tiempos es también una manera particular de vivir el espacio y territorio desde paradigmas de conocimiento occidentales. Bajo este contexto y como una de las lecciones aprendidas, reconocimos que no solo era necesario comprender la manera cómo ellas habitan el mundo colectiva y comunitariamente, sino también, diseñar con ellas las estrategias metodológicas explicando porqué para nosotras resultaba complejo entender que no era posible tener conversaciones individuales con ellas ni tener un mínimo control sobre el tiempo para nuestra planeación.

Nos enfocamos entonces, en romper con nuestras propias expectativas y planes, dejar que los tiempos se dieran. Esto significó reflexionar sobre nuestra forma de vivir el tiempo y cómo invitar a escenarios flexibles al proyecto mismo. Después de varios meses de trabajo y de conversaciones continuas, comprendimos que era importante realizar actividades que se centraran en intercambiar conocimientos y que fueran usualmente empleados por las mujeres. Por tanto, junto a las mujeres y el gobernador mayor indígena, decidimos hacer la reflexión metodológica del proyecto usando como espacio la cocina, un ritual de medicina tradicional diseñado por el Jaibaná y una creación de artesanías no-tradicionales para ellas.

En el camino, además, conversábamos como investigadoras sobre la necesidad de encontrar lenguajes comunes que nos permitieran acercarnos de manera más desprevenida a las mujeres indígenas. Desde la academia traemos un lenguaje técnico que representa un reto de-construir cuando entramos en estos espacios. Traíamos la categoría de arte en un inicio suponiendo que guardaba algún sentido con sus prácticas tradicionales, mientras que, en su lenguaje de enunciación, la referencia es a la cultura propia y no al arte la que tiene el sentido de transmisión. Fuimos comprendiendo que la transmisión no solo pasaba por la entrega de información, sino por la construcción de los sentidos, signos y significados que se derivan de aquello que se transmite. En medio de las danzas era posible entender estos significados, siendo la profesora de danzas embera el vehículo que permitía representar estos significados e interpretar lo que nosotras leíamos desde nuestras subjetividades.

\section{Encuentro 3: el giro decolonial, lecturas críticas del poder y la gestión de riesgos}

¿Como hacer sentido a lo aprendido con y desde las mujeres embera? Es en este momento que los textos de la geografía crítica feminista y la teoría decolonial nos permiten comprender lo vivido durante dos años junto a las mujeres embera, desde la reflexividad continua a la que ellas mismas nos invitaron a practicar durante nuestros encuentros. ¿De dónde vienen nuestras ideas sobre la gestión de riesgo?, ¿quién desarrolló estas ideas?, ¿qué es hacer investigación acción participativa y hacia dónde queremos dirigir nuestras prácticas de investigación?

\section{El cuidado como categoría de saber y hacer}

Autoras y autores como Fernández, Waldmüller y Vega (2020), muestran como los estudios de la reducción del riesgo de desastres se han centrado en mantener el flujo de mercado y la productividad, dejando de lado enfoques ecosistémicos que incluyan otras versiones del habitar sostenible. De igual manera, resaltan la necesidad de integrar los conocimientos, buscar un diálogo de saberes que permita entender las estrategias locales para los efectos del cambio climático. En este sentido, surge la necesidad de una conversación entre el conocimiento científico y el conocimiento de las comunidades indígenas, partiendo desde marcos diferenciados (Mercer, Kelman, Suchet-Pearson y Lloyd, 2009).

Veland, Howitt, Dominey-Howes, Thomalla y Houston (2012) enfatizan cómo la colonización de la gestión del riesgo se convierte en un continuo desastre para los pueblos indígenas, creando una necesidad epistemológica en el campo por legitimar su conocimiento. Estos mismos autores enfatizan, además, que el sentido de urgencia que ha tenido los estudios de riesgos y desastres y el cambio climático, perpetúan legados paternales y acciones verticales de poder que no logran acciones sostenibles para la comunidad.

Ante estas tensiones y necesidades, nos encontramos con el cuidado, como categoría epistémica que reconoce las formas de relacionarse con el territorio de las mujeres embera. Desde 
los estudios de la gestión del riesgo, el cuidado y la ética del cuidado -categoría derivada- han sido poco estudiados. Solamente se ha dado una aproximación a estos conceptos, desde un campo clínico que promueve una relación ética y eficiente entre paciente (damnificado) y los trabajadores de la salud, con el objetivo de garantizar y mejorar servicios de salud dignos durante y después de las emergencias (Aitsi-Selmi \& Murray, 2016).

Proponemos entender el cuidado como una "actividad y práctica o disposición moral" (Power, 2019 , p. 3) permanente direccionada hacia el bienestar de los otros, que se teje entre lo público, lo privado, lo íntimo y lo político. En este sentido, el cuidado se ubica como un bien público, un derecho fundamental y una necesidad vital para lograr la igualdad de género (CEPAL, 2016).El cuidado ha sido una categoría inicialmente profundizada por los estudios y movimientos feministas (Robinson, 2021), en la que se han desarrollado teorías de la moral con un enfoque de género (Gilligan, 1986; Noddings, 2003). Desde una perspectiva colonial y occidental, el cuidado se ha ubicado en el campo privado, reflexionando sobre la noción de familia y estado (Waitt \& Harada, 2016), así como de salud física y mental (Groot, Haveman \& Abma, 2020).

Sin embargo, estudios críticos recientes de las ciencias sociales han señalado las consecuencias de centrarse solo en el ámbito privado. Quiroga (2011) afirma que esto ha excluido nuestro contexto regional y étnico dentro de América Latina y ha resultado en una falta de reconocimiento de las formas de cuidado colectivas y comunitarias. Además, recientemente se han incrementado las investigaciones en torno al cuidado, la justicia social y las emociones (Conradson, 2003). Estos estudios críticos han demostrado la alta desigualdad entre mujeres y hombres en la participación en el mercado y el compromiso ciudadano a través de la economía y las políticas del cuidado (Light \& Akama, 2014). Asimismo, la geografía crítica también ha logrado posicionar algunas reflexiones sobre lo que implica el cuidado del espacio en relación con el cuerpo afirmando que el cuidado en sí mismo es una acción de responsabilidad para el bienestar y la justicia ambiental (Lawson, 2008).

A partir de los aportes de Quiroga (2011), Power (2019) y la CEPAL (2016) entendemos que las prácticas de cuidado de las mujeres embera se derivan, no sólo de formas colectiva y comunitarias de construir realidad, además, de una relación inseparable entre cuerpo y territorio, se convierten en un mismo elemento. Al cuidar el territorio, a través de la medicina tradicional y los rituales, se cuidan colectivamente a los cuerpos. La sanación del territorio es la sanación misma del cuerpo y prevenir una emergencia o restaurar el equilibro de los jais después de un evento es también prevenir la enfermedad y/o curar. "(...) Lo que queremos decir es que se puede prevenir a tiempo el desastre y la enfermedad de las familias indígenas (...) lo que estamos haciendo es previniendo el desastre el que va venir, como medicina (...)" (Gobernador Mayor, Cabildo Indígena Kurmadó, 27 de octubre 2018).

Los aprendizajes evocan entonces dos posiciones de cuidado. El cuidado territorial en el que está implicado el cuerpo como territorio en sí mismo, como una extensión del espacio (Zaragocin y Caretta, 2020), más no como un instrumento de ella; y el cuidado relacional -a nivel metodológicoque se prefigura en el vínculo tejido entre las mujeres, entre ellas y el Jaibaná, entre ellas y nosotras como investigadoras.

Es importante aclarar que la lectura del cuidado que realizamos aquí no hace referencia a una versión ideal creada e impuesta sobre las comunidades indígenas del deber ser con el territorio y su relación con la naturaleza. Esta relación de cuidado y reciprocidad no implica una condición inmanente de armonía en las maneras de habitar y vivir el territorio de las comunidades indígenas. Es decir, valorar y aprender de estas ontologías no significa imponer a las comunidades una percepción o cercanía a la naturaleza mayor o menor a otros grupos comunitarios.

Partimos desde una visión crítica y experiencial sobre las prácticas y concepciones de cuidado de las mujeres embera hacia y desde sus cuerpos, sus hogares y sus territorios. Seres movilizados por los médicos tradicionales a través de rituales, como el Benecua, tienen el poder de propiciar emergencias y desastres; sin embargo, son los cuerpos los principales receptores del daño y, por ende, responsables del cuidado del territorio. El médico tradicional tiene un rol clave para la 'reducción del riesgo de desastres', es cuidador -motor clave de un territorio seguro-, y a su vez, es amenaza. "El Jaibaná en cada sector es el que aconseja, cuando llueve se les dice: - no salga, no saque a los niños que esos espíritus están por ahí sueltos (...)" (Médico tradicional embera, 27 de octubre 2018). Esto mismo sucede con el conocimiento hacia las plantas medicinales. 
Si el medico tradicional es quien tiene la capacidad de comprender y cuidar esta relación, es importante que él pueda acompañar y liderar desde su saber los riesgos que las comunidades perciben en sus territorios. Si esto no se tiene en cuenta, no sabremos definir rutas metodológicas con las comunidades, ni será posible la coproducción de conocimiento.

Estas reflexiones invitan a los estudios de riesgos y desastres a problematizar algunos de sus fundamentos teóricos para leer el territorio de manera distinta. Proponemos desligarnos, por un lado, de la idea de amenaza natural por un momento. Para las mujeres embera, la amenaza no es natural, es creada por Jais -seres milenarios-, y al mismo tiempo puede desaparecer 0 irse a otro lugar a través de la intervención de los médicos tradicionales y el uso de plantas medicinales por parte de la comunidad. Por otro lado, proponemos alejarnos del ideal occidental de poder controlar la naturaleza y reducir la incertidumbre, a través de la apropiación y gestión de ella (Walsh 2015). La relación del pueblo embera con los jais y el territorio nos interpela a pensar y sentir en otras posibilidades de relacionarse con y en el territorio. El cuidado y reciprocidad colectiva con los jais que a su vez significa el cuidado colectivo de las personas así como de las personas con el territorio, nos abre puertas a pensar en una existencia plural que no se encuentre fundamentada en la dualidad naturaleza - seres humanos ni en un ideal de apropiación y control sobre la naturaleza basado en el pensamiento occidental (Walsh 2015).

Es justamente a partir del cuidado que entendemos esta relación no lineal entre los cuerpos colectivos y el territorio y llegamos a lo que propone la perspectiva decolonial, "reatar con lo que existe, con los jirones de comunidad que continúan su camino histórico (...) Abrir brechas, fisuras en el tejido estabilizado de la colonial modernidad, y dar camino de continuidad a los proyectos históricos de los pueblos" (Segato, 2018a, p.64). Desde esta perspectiva, se abren críticas a la modernidad que invitan a una desobediencia epistémica (Mignolo, 2010), y por ende, invita a ubicarse desde otras formas de ser y estar en el territorio. Nos alejamos de las maneras cómo se "organiza el mundo ontológicamente en términos de categorías homogéneas, atómicas, separables" (Lugones, 2011, p.106). La categoría de cuidado nos invita a una relación inseparable con las formas que protegemos y nos autoprotegemos, marcando un ritmo el cual no es controlado por un plan dependiente a una fecha, un día o una hora.

Necesitamos develar cómo desde el pensamiento occidental, lo moderno se torna en un ideal que pretende homogeneizar como único deseo en la sociedad, cobrando un valor superior bajo una "jerarquía fundacional, basada y construida sobre el cimiento de la raza" (Segato, 2018b, p.48). Es a partir de esta jerarquía que los saberes de los pueblos indígenas "pasan a regirse por ese escalafón de prestigio" (Segato, 2018b, p.50) y bajo categorías supuestamente neutrales, objetivas y universales; situación que configura un racismo epistémico, en cuanto "discrimina saberes y producciones, reduce civilizaciones, valores, capacidades, reacciones y creencias" (Segato, 2018b, p. 53).

La ética del cuidado como práctica decolonial nos invita a preguntarnos, ¿debemos integrar los conocimientos o debemos aprender a ubicarnos desde otras formas de pensamiento para construir ciencia? La gestión del riesgo de desastres, no ha dado cuenta de las formas plurales de vivir y entender el riesgo, de vivir según un proyecto histórico "(...) donde A y no-A pueden ser verdades al mismo tiempo y no se excluyen" (Segato, 2018a, p.100). Si un fenómeno se ubica como amenaza se convierte en $\mathrm{A}$, lo que hace que la fórmula impida en sí misma comprender verdades y relatos simultáneos que no impliquen discrepancia y separación.

Esto también se evidencia en lo que hemos descrito como nuestro primer desencuentro sociolingüístico. Desde la lengua embera no hay interpretación para ambas palabras, no existe. La gestión del riesgo nace y cobra sentido en el campo de las finanzas y ambientes empresariales (enterprise risk management) (Ruiz, Jiménez y Torres, 2000) y riesgo en el pensamiento moderno occidental (Beck, 1992). Este vacío semántico revela la problemática-ontológica-colonial que tiene en sí misma la gestión del riesgo de desastres. Justamente, es este llamado el que nos interpela para responder a la pluralidad y modos diversos de la construcción territorial, problematizando la interpretación del riesgo que introduce la fórmula y subsecuentemente la gestión de riesgos de desastres.

\section{Voces plurales desde la reciprocidad}

Acercarnos al giro decolonial, también nos ha permitido ser interpeladas por preguntas alrededor de quién y para qué se construye el conocimiento desde los estudios de riesgos y como 
tal de la gestión del riesgo de desastres. Estas preguntas, nos han permitido notar que las ideas y el conocimiento que se encuentra actualmente más citado en el campo de la gestión del riesgo de desastres, está no solamente construido desde epistemologías occidentales sino también, es mayoritariamente construido desde las voces masculinas (Maskrey, 1993; Wilches-Chaux 1993; Lavell, 2001; Wisner et. al 2004; Cardona, 2008; Wisner, Gaillard y Kelman, 2011; Alexander, 2013; Shaw, 2014). Al igual que las jerarquías impuestas alrededor del conocimiento, el giro decolonial también explica cómo en el escalafón de prestigio de la modernidad "el lugar de las mujeres, muy en especial de las mujeres de razas inferiores, quedó estereotipado junto el resto de los cuerpos, y tanto más inferiores fueran sus razas, tanto más cerca de la naturaleza [...]" (Segato, 2018b, p. 54). Nos preguntamos entonces: ¿Cuáles son las jerarquías de poder y conocimiento que rigen la 'ciencia' de los riesgos y desastres? ¿Cómo podemos incluir y visibilizar voces plurales y el conocimiento femenino al legado mayoritariamente masculino en los estudios de desastres expuesta en nuestro primer encuentro? ¿Cómo podemos romper con este 'escalafón de prestigio' y cuál es lugar de los saberes de las mujeres indígenas embera?

A partir de estas reflexiones también ubicamos el cuidado, como categoría que nace de las experiencias entre voces femeninas. No obstante, emerge una segunda categoría la cual convoca paralelamente lo conceptual y metodológico: la reciprocidad. Si bien tiene íntima relación con el cuidado, nos permite materializar con mayor facilidad los enfoques metodológicos. Davis (2015, p.106) enfatiza que la reciprocidad "(..) define y regula la conexión que se establece entre la comunidad y la tierra, obligaciones y relaciones rituales que no se expresan de manera verbal". Así, el cuidado y la reciprocidad involucran una mirada ontológica del otro como agente en su territorio, en la medida que construye relación y busca salvaguardar la vida en relación con los otros. Silvia Rivera Cusicanqui (2018) nos invita a mirar la reciprocidad como un epítome de lo social basado en la capacidad de intercambio con el mundo que nos rodea. Es justamente esa capacidad la que se convoca a la hora de realizar los rituales Benecua o las formas de cuidado de las mujeres tienen con sus cuerpos, los de sus familias y sus territorios.

El cuidado y la reciprocidad también nos involucra a nosotras como investigadoras; referencias como "[...] nos gusta que nos digan lo que no les gusta [...]" y "(...) desde el principio hasta el final, no que solamente vino por su papel, por su foto [...]" (Mujer embera, citada en Armijos, López y Ramírez, 2018), pone en tensión relaciones pasivas en las que ni las y los investigadores, ni las comunidades, se exponen. La reciprocidad, particularmente, nos permite entender el tiempo como permanencia (Armijos, López y Ramírez, 2018). El acompañamiento a las mujeres, se hace posible en la medida que ellas participan en el proyecto, y por ende, co-construyen conocimiento con los investigadores. Eso es lo que llamamos tiempo de reciprocidad. Las conversaciones se volvieron espacios de intercambio de saberes, no solamente entre las mujeres emberas y el equipo de investigadores, si no también fue un espacio de diálogo y aprendizaje intergeneracional y territorial. Según una joven indígena estos espacios: [sirvió mucha (mucho) porque esas conversaciones son para nosotras, como eran los antiguos, entonces nosotros tenemos que estar también como recordando[...] (Comunicación personal, Caimalito, 25 de octubre de 2018).

\section{CONCLUSIÓN: CAMINAMOS HACIA NUEVOS DESENCUENTROS, LA RECIPROCIDAD Y EL CUIDADO COMO PRACTICAS ÉTICO POLÍTICAS PARA LOS ESTUDIOS DE RIESGOS Y DESASTRES}

La fórmula creada desde el enfoque de las ciencias aplicadas, ha derivado en un sinnúmero de estudios desde las ciencias sociales, como lo refleja la revisión de literatura y estado del arte hecho por Alexander et al. (2020). Sin embargo, una vez interpeladas por estos desencuentros con la gestión del riesgo, la fórmula y los estudios de riesgos y desastres mayormente posicionados desde el pensamiento occidental y masculino, nos impiden otro tipo de comprensiones, entendimientos que parten de la circularidad y que quebrantan el pensamiento binario el cual, desde la fórmula, se refleja en las oposiciones que evoca: amenaza-seguridad o prevención-recuperación. Este binarismo resulta complejo de traducirse, interpretarse y entenderse por parte de las mujeres embera. Su episteme -forma de conocer la realidad-, no se ubica desde esta linealidad, lo que crea otro tipo de responsabilidades y sentires a lo que significa sentirse en peligro 0 ante un evento que, para el conocimiento científico del mestizo, resulta ser problemático. Así es como la fórmula se impide a sí misma reconocer aquel espíritu trágico que connota la vida, la incertidumbre misma, como nos plantea Segato (2018a) y que desde la cultura occidental, es enseñada desde el olvido 
en el transcurrir de la vida, o desde el negativo-positivo que media la dualidad tanto en el cuerpo como en los espacios geográficos.

En este sentido, tanto el cuidado como la reciprocidad ponen en tensión temas que actualmente, para la "gestión del riesgo de desastres", son una prioridad innovadora y trascendente, pensadas desde el mestizo, el blanco o el criollo, como lo diría Segato (2018a). Entre estos temas se encuentra la incertidumbre, preocupación central para la cultura occidental que, sin embargo, parece ser una cuestión resuelta desde otros lugares epistémicos, en cuanto la predictibilidad no hace parte de sus proyectos históricos como comunidad ni como mujeres indígenas.

El cuidado nos ha permitido mirar a la periferia para encontrar otros caminos que nos permitan entender mejor el desencuentro sociolingüístico con el riesgo. Si el colonialismo define las relaciones intersubjetivas, nuestras formas de pensamiento, nuestro lenguaje (MaldonadoTorres, 2008), el riesgo se convierte en una categoría colonial que, más allá de pretender ser anulada o borrada, debe hacer un llamado a la ética insatisfecha (Segato, 2018a) la cual convoca a la sospecha sobre la mirada propia y ajena, para oponernos a la muerte del Otro y dar apertura a la generosidad (Maldonado-Torres, 2008). Esta generosidad, entonces, nos ubica en la colectividad como forma de pensamiento, no solo como intencionalidad de agrupación, hecho que para la linealidad y mirada binaria y dualista de la fórmula y del pensamiento occidental derivado de ella, resulta ser incomprensible.

La reciprocidad involucra las relaciones internas de la comunidad, visibilizando la relación que se teje entre géneros, la relación con las y los investigadores, y la relación con las organizaciones/ entidades/instituciones. Sin embargo, la reciprocidad también involucra el encuentro de cuerposterritorios que se sitúan como sujetos activos de conocimiento. Es justamente aquí donde entra el giro decolonial y en consonancia, las metodologías participativas. Estos vínculos y encuentros nos abren las puertas hacia otras prácticas de investigación, más aún en los estudios de riesgo y desastres; practicar el conocimiento situado (Haraway 1991) y el "pensamiento crítico de frontera" (Cuevas, 2013, p.83) que permitan definir estrategias metodológicas transdisciplinares junto con las comunidades y los gobiernos locales.

Regresamos a la pregunta insatisfecha: ¿qué puede (o debe) aprender los estudios de riesgos y desastres de estas mujeres y de sus saberes del habitar el territorio? El cuidado de los territorios. Tanto el cuidado como la reciprocidad terminan siendo categorías que nos interpelan en nuestro quehacer investigativo, llegan a nuestra subjetividad para cuestionarnos, para convertirnos en objetos de conocimiento; es desde allí que nace la reciprocidad, desde el lugar en el que también comunicamos nuestras prácticas culturales y nuestras reflexiones. De ahí que nuestros relatos autobiográficos brindan apertura a la relación con las mujeres embera y a la narrativa escrita partiendo desde nuestros cuerpos-territorios.

\section{AGRADECIMIENTOS}

Queremos agradecer a todas las mujeres que fueron parte de este camino, especialmente a las gobernadoras menores con quienes buscamos formas de tejer vínculos basados en la confianza y el respeto por la palabra de cada una. Asimismo, agradecemos al equipo de la Universidad de Manizales y de la Universidad de East Anglia, sin cada una y uno de ustedes no hubieran sido posibles los espacios colaborativos construidos con las comunidades: Lina Andrea Zambrano Hernández, Alejandra López Getial, Roger Few, Hazel Marsh, Andrés Carvajal Díaz, Tatiana Valencia Serna, Santiago Urrea Yela, Viviana Paola Grajales Vargas, Valentina Bedoya Cadavid, Miguel Ángel Hernández Ramírez, Giovanny Gaitán Arias, Laura Manuela Mozo Vélez y Luis David Acosta Rodriguez. Por último, agradecemos al Consejo de Investigación de Artes y Humanidades del Reino Unido (AHRC) por haber confiado en nuestro trabajo y continuar con el proceso de pensar otras formas de investigación que nos lleven a un trabajo participativo basado en un diálogo de saberes.

\section{REFERENCIAS}

Alexander, D. (2013). Resilience and disaster risk reduction: an etymological journey. Nat. Hazards

Earth Syst. Sci., 13(11), 2707-2716. https://doi.org/10.5194/nhess-13-2707-2013

Alexander, D., Gaillard, J.C., Kelman, I., Marincioni, F., Penning-Rowsell, E., Niekerk, L. \& Vinnell, L.J.

(2020). Academic publishing in disaster risk reduction: past, present, and future. Disasters, 46(1).

1-14. https://10.1111/disa.12432 
Alcaldía de Pereira. (2011). Plan de vida comunidad indígena asentada en el municipio de Pereira. http://www.pereira.gov.co/Transparencia/poblacin\%2ovulnerable/Acuerdo_No_56_Plan_de_vida_ comunidad_indigena_asentada_en_el_municipio_de_Pereira.pdf

Altamirano, C.A. \& Sanchez, Y.A. (2017). Juegos autóctonos, tradicionales y modernos del Cabildo Indígena de Kurmadó (trabajo de grado). Universidad Tecnológica de Pereira.

Aitsi-Selmi A \& Murray V. (2016). Protecting the health and well-being of populations from disasters: health and health care in The Sendai Framework for Disaster Risk Reduction 2015-2030. Prehosp Disaster Med., 31(1), 74-78.

Armijos M.T., Lopez Geital, A., \& Ramirez Loaiza, V. (2020). Indigenous engagement, research partnerships, and knowledge mobilization. AHRC. https://webarchive.nationalarchives.gov. uk/20200531032105/https://www.ukri.org/news/esrc-ahrc-gcrf-indigenous-engagement-programme/

Beck, U. (1992). Risk Society: Towards a New Modernity (traducido por Mark Ritter). Sage Publications.

Bender, S. (1993). Preparación en caso de desastres y desarrollo sostenible. Desastres E Sociedad, (1), 98-102.

Cardona, O.D. (2008). Medición de la gestión del riesgo en América Latina. Revista Internacional de Sostenibilidad, Tecnología y Humanismo, (3), 1-20. https://upcommons.upc.edu/handle/2099/7056

Conradson, D. (2003). Geographies of care: spaces, practices, experiences. Social ECultural Geography, $4(4), 451-454$.

Cuevas, P. (2013). Memoria colectiva: hacia un proyecto decolonial. En C. Walsh (ed.). Pedagogías decoloniales. Prácticas insurgentes de resistir, (re)existir y (re)vivir (tomo I). Abya-Yala.

Cruz, M. A., Reyes, M. J. \& Cornejo, M. (2012). Conocimiento Situado y el Problema de la Subjetividad del Investigador/a. Cinta de moebio, (45), 253-274. https://dx.doi.org/10.4067/ So717-554X2012000300005

CEPAL. (2016). Políticas de cuidado en América Latina Forjando la igualdad. Serie Asuntos de Género, (140), Naciones Unidas.

Davis, W. (2015). Los guardianes de la sabiduría ancestral. Su importancia en el mundo moderno. Sílaba Editores.

Evans, V.J. (1994). Percepción del riesgo y noción de tiempo. Desastres E Sociedad, (3), 9-22.

Fals Borda, O. (2009). La investigación acción en convergencias disciplinarias. Revista Paca, (1), 7-21. https://doi.org/10.25054/2027257X.2194

Fernández, A. G., Waldmüller, J. \& Vega, C. (2020). Comunidad, vulnerabilidad y reproducción en condiciones de desastre. Abordajes desde América Latina y el Caribe. Íconos. Revista de Ciencias Sociales, (66), 7-29. https://doi.org/10.17141/iconos.66.2020.4156

Few, R., Ramírez, V., Armijos, M.T., Zambrano, L.A. \& Marsh, H. (2021). Moving with risk: Forced displacement and vulnerability to hazards in Colombia. World Development, 144. https://doi. org/10.1016/j.worlddev.2021.105482

García, V. (2005). El riesgo como construcción social y la construcción social de riesgos. Desacatos, (19), 11-24. http://www.scielo.org.mx/pdf/desacatos/n19/n19a2.pdf

García, V. (2017). Divinidad y desastres. Interpretaciones, manifestaciones y respuestas. Revista de Historia Moderna, (35), 46-82. http://10.14198/RHM2017.35.02

Gilligan, C. (1986). Moral orientation and moral development. In: E. F. Kittay \& D. T. Meyers (Eds) Women and Moral Theory, 19-33. Rowman \& Littlefield.

Groot, B., Haveman, A. \& Abma, T. (2020). Relational, ethically sound co-production in mental health care research: epistemic injustice and the need for an ethics of care. Critical Public Health. https:// doi.org/10.1080/09581596.2020.1770694

Haraway, D. (1991). Ciencia, cyborgs y mujeres: la reinvención de la naturaleza. Ediciones Cátedra.

LA RED. (1993). Editorial. Desastres E Sociedad, (1), 3-5.

LA RED. (1994). Editorial. Desastres E Sociedad, (3), 5-6.

Lavell, A. (2001). Sobre la gestión del riesgo: apuntes hacia una definición. Biblioteca Virtual en Salud de Desastres. https://www.undp.org/content/dam/undp/documents/cpr/disred/espanol_/glr_andino/ docs/METODOLOGIA\%2oDE\%2oSISTEMATIZACI\%C3\%93N\%2oPARA\%2oDIAGRAMAR/apuntes hacia_una_definici_n_de_la_gesti_n_de_riesgo_A_llan_Lavell.pdf 
Lawson, V. (2007). Geographies of Care and Responsibility. Annals of the Association of American Geographers, 97(1), 1-11. https://10.1111/j.1467-8306.2007.00520

Light, A. \& Akama, Y. (2014). Structuring future social relations: the politics of care in participatory practice. Proceedings of the 13th Participatory Design Conference: Research Papers, 1, 151-16o.

Lugones, M. (2011). Hacia un feminismo descolonial. La manzana de la discordia, 6(2), 105-119.

Maldonado-Torres, N. (2008). La descolonización y el giro des-colonial. Tabula Rasa, 9, 61-72.

Marsh, H., Armijos M.T., \& Few, R. (2020). Telling it in our own way: Doing music-enhanced interviews with people displaced by violence in Colombia. New Area Studies, 1(1), 132-164.

Maskrey, A. (1998). Capítulo 1: El riesgo. En: A. Maskrey (ed.). Navegando entre brumas. La aplicación de los sistemas de información geográfica al análisis de riesgos en América Latina, pp.9-3o. ITDG/LA RED.

Maskrey, A. (comp.). (1993). Los desastres no son naturales. LA RED. https://www.desenredando.org/ public/libros/1993/ldnsn/

Mercer, J., Kelman, I., Suchet-Pearson, S. \& Lloyd K. (2009). Integrating Indigenous and Scientific Knowledge Bases for Disaster Risk Reduction in Papua New Guinea. Geografiska Annaler. Series B, Human Geography, 91(2), 157-183.

Mignolo, W. (2010). Desobediencia epistémica. Retórica de la modernidad, lógica de la colonialidad y gramática de la descolonialidad. Ediciones del Signo.

Narvaez, L. Lavell, A. \& Pérez, G. (2009). La gestión del riesgo de desastres; un enfoque basado en procesos. Maiteé Flores Piérola - PULL CREATIVO S.R.L

Noddings, N. (2003). Caring: A Feminine Approach to Ethics E Moral Education. University of California Press.

Power, E. (2019). Assembling the capacity to care: Caring-with precarious housing. TIBG, 44(4), 763-777. https://doi.org/10.1111/tran.12306

Quiroga, N. (2011). Economía del cuidado. Reflexiones para un feminismo decolonial. Rev. Casa de la Mujer, 20(2), 97-116.

Robinson, F. (2021). Feminist foreign policy as ethical foreign policy? A care ethics perspective. Journal of International Political Theory,17(1), 20-37. https://doi.org/10.1177/1755088219828768

Ruiz, G., Jiménez, J.I. \& Torres, J.J. (200o). La gestión del riesgo financiero. Ediciones Pirámide.

Shaw, R. (2014). Community Practices for Disaster Risk Reduction in Japan. Springer.

Segato, R. (2018a). Contra-pedagogías de la crueldad (1era Ed.). Prometeo Libros.

Segato, R. (2018b). La crítica de la colonialidad en ocho ensayos y una antropología por demanda. Prometeo Libros.

Turner, B.L., Kasperson, R.E., Matson, P. Mccarthy, J.J., Corell, R.W., Christensen, L., Eckley, N., Kasperson, J.X., Luers, A., Martello, M.L., Polsky, C., Pulsipher, A. \& Schiller, A. (2003). A framework for vulnerability analysis in sustainability science. Proceedings of the National Academy of Sciences of the United States of America, 100(14). 8074-8079. https://groups.nceas.ucsb.edu/sustainabilityscience/2010\%2oweekly-sessions/session-3-09.27.2010/supplemental-readings-from-princetongroup/Turner\%20et\%202003\%20Vulnerability\%2oframework.pdf/at_download/file

Rivera Cusicanqui, S. (2018). Un mundo ch’ixi es posible. Ensayos desde un presente en crisis. Tinta Limón.

Veland, S., Howitt, R., Dominey-Howes, D., Thomalla, F. \& Houston, D. (2013). Procedural vulnerability: Understanding environmental change in a remote indigenous community. Global Environmental Change, 23(1), 314-326.

Walsh, C. (2013). Pedagogías decoloniales. Prácticas insurgentes de resistir, (re)existir y (re)vivir (Tomo 1). Serie Pensamiento Decolonial.

Walsh, C. (2015). Life, nature and gender otherwise: feminist reflections and provocations from the Andes. En Harcourt, W. \& Nelson, I.L. (eds), Practising Feminist Political Ecologies: Moving Beyond the Green Economy. Zed Books.

Waitt, G., \& Harada, T. (2016). Parenting, care and the family car. Social \& Cultural Geography, 17, 1079-110o. https://doi.org/10.1080/14649365.2016.1152395 
Wilches-Chaux, G. (1993). La vulnerabilidad Global. En Maskrey, A. (Ed.), Los Desastres no son Naturales. La RED/Tercer Mundo Editores.

Wilches-Chaux, G. (2017). El concepto-herramienta de la seguridad territorial y la gestión de humedales. Biodiversidad En La Práctica, 2(1), 48-86. http://revistas.humboldt.org.co/index.php/ $\mathrm{BEP} /$ article/view/453

Wisner, B., Balikie, P., Cannon, T. \& Davis, I. (1996). At Risk. Natural Hazards, people's vulnerability and disasters. Routledge.

Wisner, B., Balikie, P., Cannon, T. \& Davis, I. (2004). At Risk. Natural Hazards, people's vulnerability and disasters (2nd Ed.). Routledge.

Wisner, B., Gaillard, J.C. \& Kelman, I. (eds). (2011). Handbook of Hazards and Disaster Risk Reduction. Routledge.

Zaragocin S \& Caretta, M.A. (2020). Cuerpo-Territorio: A Decolonial Feminist Geographical Method for the Study of Embodiment. Annals of the American Association of Geographers, 11(5), 1503-1518. https://10.1080/24694452.2020.1812370 